PROCEEDINGS OF THE

AMERICAN MATHEMATICAL SOCIETY

Volume 131, Number 7, Pages 2201-2207

S 0002-9939(02)06793-X

Article electronically published on November 6, 2002

\title{
GENERALIZED TCHAKALOFF'S THEOREM FOR SEMI-SPECTRAL MEASURES
}

\author{
LIH-CHUNG WANG AND CHIH-RUNG CHEN
}

(Communicated by Joseph A. Ball)

\begin{abstract}
We proved the existence of exact quadrature formulae with semipositive definite coefficient matrices for polynomials of prescribed degree in $n$ variables and with respect to a semi-spectral measure. Our proof could be viewed as a direct translation (generalization) of Putinar's result on the existence of quadrature formulae for a positive measure without compact support.
\end{abstract}

\section{INTRODUCTION}

The classical one-dimensional moment problem could be successfully answered by several approaches. For the higher dimensional case, there is much less known information. The theory of quadrature identities is one of the main themes. Tchakaloff's Theorem [5] asserts the existence of an exact quadrature formula with positive coefficients for polynomials of prescribed degree in $n$ real variables and with respect to a positive measure with compact support which is absolutely continuous with respect to Lebesgue $n$-volume measure. This result was the source of several further developments in the theory of quadrature formulas; cf. [4.

We generalize Tchakaloff's Theorem to arbitrary semi-spectral measure in the Euclidean space. The motivation for this investigation came from the study of semispectral measure for the control theory and from some interesting work of Curto and Fialkow on truncated multi-variable moment problems during the discussion with M. Putinar. We believe that people who study linear control theory may be interested in quadrature problems for matrix-valued measures.

Let $x=\left(x_{1}, \ldots, x_{n}\right)$ be the current vector in $\mathbb{R}^{n}$ and let $P_{d}\left(\mathbb{R}^{n}\right)$ be the real vector space of polynomials in $x$ of total degree less than or equal to $d$. Let $N_{d}\left(\mathbb{R}^{n}\right)$ be the dimension of $P_{d}\left(\mathbb{R}^{n}\right)$. We say that $E$ is a semi-spectral measure if $E(X)$ is a symmetric semi-positive definite $(l \times l)$ matrix for an arbitrary measurable set $X \subset \mathbb{R}^{n}$. Our main results are stated in the following two theorems.

Theorem 1. Let $E$ be a semi-spectral measure with compact support $K$ in $\mathbb{R}^{n}$ and let $d$ be a fixed positive integer. Then there are $N\left(\leq l^{2} N_{d}\left(\mathbb{R}^{n}\right)\right)$ points $x_{j} \in K$ and

Received by the editors March 1, 2001 and, in revised form, February 12, 2002.

2000 Mathematics Subject Classification. Primary 65D32, 44A60.

This paper was partially supported by the National Science Council (NSC-87-2119-M-259-004). 
semi-positive definite matrices $A_{j}, j=1, \ldots, N$, such that

$$
\int_{\mathbb{R}^{n}} p d E=\sum_{j=1}^{N} A_{j} p\left(x_{j}\right)
$$

for all $p \in P_{d}\left(\mathbb{R}^{n}\right)$.

Note that we may replace $l^{2} N_{d}\left(\mathbb{R}^{n}\right)$ by the smaller upper bound $\frac{l(l+1)}{2} N_{d}\left(\mathbb{R}^{n}\right)$ in the above assumption.

Theorem 2. Let $d$ be a fixed positive integer and let $E$ be a semi-spectral measure in $\mathbb{R}^{n}$ with the property that

$$
\int_{\mathbb{R}^{n}}\left|x^{\alpha}\right| d E
$$

exists for every multi-index $\alpha$ such that $|\alpha| \leq 2 d$, where $\alpha=\left(\alpha_{1}, \alpha_{2}, \ldots, \alpha_{n}\right)$, $x^{\alpha}=x_{1}^{\alpha_{1}} x_{2}^{\alpha_{1}} \cdots x_{n}^{\alpha_{n}}$ and $|\alpha|=\alpha_{1}+\alpha_{2}+\ldots+\alpha_{n}$. Then there are $N\left(\leq l^{2} N_{2 d}\left(\mathbb{R}^{n}\right)\right)$ points $x_{j} \in \operatorname{supp}(E)$ and semi-positive definite matrices $A_{j}, j=1, \ldots, N$, such that

$$
\int_{\mathbb{R}^{n}} p d E=\sum_{j=1}^{N} A_{j} p\left(x_{j}\right)
$$

for all $p \in P_{2 d-1}\left(\mathbb{R}^{n}\right)$.

Trivially, we have the following corollary.

Corollary 3. Let d be a fixed positive integer and let $E$ be a semi-spectral measure in $\mathbb{R}^{n}$ with the property that

$$
\int_{\mathbb{R}^{n}}|p| d E
$$

exists for every $p \in P_{2 d}\left(\mathbb{R}^{n}\right)$. Then the conclusion in Theorem 2 holds.

If the support of the semi-spectral measure is contained in a proper closed convex cone of $\mathbb{R}^{n}$ generated by $n$ linearly independent vectors, we may assume that the support of the semi-spectral measure is in the first octant $\mathbb{R}_{+}^{n}$ with an affine transformation. If the moments of $E$ up to degree $d$ exist, both the condition in Theorem 2 and the positivity needed in the proof of Theorem 2 are trivially satisfied. Hence we have the following corollary.

Corollary 4. Let $E$ be a semi-spectral measure in $\mathbb{R}^{n}$ with support contained in a proper convex cone and with moments up to degree $d$. Then there are $N(\leq$ $\left.l^{2} N_{2 d}\left(\mathbb{R}^{n}\right)\right)$ points $x_{j} \in \operatorname{supp}(E)$ and semi-positive definite matrices $A_{j}, j=1, \ldots$, $N$, such that

$$
\int_{\mathbb{R}^{n}} p d E=\sum_{j=1}^{N} A_{j} p\left(x_{j}\right)
$$

for all $p \in P_{d-1}\left(\mathbb{R}^{n}\right)$.

Note that the degree in Corollary 4 need not be even as in Theorem 2 since the positivity needed in the proof is from the support of $E$ in the first octant and not from the top degree any more.

In the theory of quadrature identities, finding the quadrature formulae with a minimal number of nodes (i.e., finding so-called Gaussian quadratures) is a very important subject. We hope to return to this matter elsewhere. 


\section{Proof of the theorems}

Our proof of Theorems 1 and 2 closely follows the method of Putinar [3].

Proof of Theorem 1. First, it is obvious that both sides of the quadrature formulae are linear. Hence, if there exist quadrature formulae for monomials in $P_{d}\left(\mathbb{R}^{n}\right)$, then these quadrature formulae are true for all polynomials $p \in P_{d}\left(\mathbb{R}^{n}\right)$. We consider the vectors

$$
v(x)=\left(1, x_{1}, x_{2}, \ldots, x_{n}, x_{1}^{2}, x_{1} x_{2}, \ldots, x_{n}^{d}\right) \in \mathbb{R}^{M}
$$

which enumerate in a prescribed order all monomials of total degree less than or equal to $d$. To simplify notation we put $M=N_{d}\left(\mathbb{R}^{n}\right)$ and $N=l^{2} M$. Clearly, $v: K \rightarrow \mathbb{R}^{M}$ gives an embedding of $K$ in $\mathbb{R}^{M}$. Define $V(K)$ to be the image of $v$, i.e.,

$$
V(K)=\{v(x) \mid x \in K\} .
$$

Since $K$ is compact, it follows that $V(K)$ is also compact in $\mathbb{R}^{M}$. Define the convex cone $C_{N}(K)$ to be the set of vectors $u \in \mathbb{R}^{l^{2} M}$ which are linear combinations

$$
u=\sum_{i} A_{i} v_{i}=\left(\sum_{i} A_{i}, \sum_{i} A_{i} x_{i 1}, \sum_{i} A_{i} x_{i 2}, \ldots, \sum_{i} A_{i} x_{i n}^{d}\right),
$$

with semi-positive definite matrices $A_{i}$, of at most $N\left(=l^{2} M\right)$ vectors $v_{i}$ of $V(K)$. Note that $v_{i}=v\left(x_{i}\right)=\left(1, x_{i 1}, x_{i 2}, \ldots, x_{i n}^{d}\right)$ for some $x_{i}=\left(x_{i 1}, x_{i 2}, \ldots, x_{i n}\right) \in K$.

In order to prove the theorem, we need to show that $C_{N}(K)$ is a closed convex cone. That is, given a sequence $\left\{u_{j}\right\}$ of vectors in $C_{N}(K)$,

$$
u_{j}=\sum_{i} A_{i j} v_{i j}
$$

which converges to a vector $u_{0}=\left(A_{01}, A_{02}, \ldots, A_{0 M}\right) \in \mathbb{R}^{l^{2} M}$, we need to show that $u_{0} \in C_{N}(K)$. The key step is to show that $\left\{\left\|A_{i j}\right\|\right\}$ is bounded, where $\left\|A_{i j}\right\|$ is the maximum of absolute eigenvalues of $A_{i j}$. Consider the first component of $u_{j}$, which is $\sum_{i} A_{i j}$ since the first component of any $v_{i j}$ is $1 . \sum_{i} A_{i j}$ converges to $A_{01}$. Hence, $\left\{\sum_{i} A_{i j}\right\}$ is bounded. The trace norm of a symmetric matrix $A$ is defined to be

$$
\sum_{\lambda: \text { eigenvalue }} \operatorname{mul}(\lambda)|\lambda|
$$

where $\operatorname{mul}(\lambda)$ is the algebraic multiplicity of $\lambda$ in $A$ (see the Appendix). Because of the semi-positivity of $A_{i j}, \sum A_{i j}$ is semi-positive definite. Since the trace norm of a semi-positive definite matrix is exactly the same as its trace, it follows that

$$
\left\|A_{i j}\right\| \leq \operatorname{Trace}\left(A_{i j}\right) \leq \sum_{i} \operatorname{Tr}\left(A_{i j}\right)=\operatorname{Tr}\left(\sum_{i} A_{i j}\right) .
$$

Since $\left\{\sum_{i} A_{i j}\right\}$ is bounded, $\left\{\left\|A_{i j}\right\|\right\}$ is bounded. Both the boundedness of $\left\{\left\|A_{i j}\right\|\right\}$ and the compactness of $V(K)$ imply that the sequence $\left\{u_{j}\right\}$ is in a compact subset of $C_{N}(K)$. Therefore, $u_{0} \in C_{N}(K)$ and $C_{N}(K)$ is a closed convex cone.

By Caratheodory's Theorem, any finite sum $\sum_{i} A_{i} v_{i}$ can be replaced by a sum of at most $l^{2} M$ terms. Let $E$ be a semi-spectral measure with compact support $K$ in $\mathbb{R}^{n}$. Since the integral $\int_{\mathbb{R}^{n}} v(x) d E(x)$ can be viewed as a limit of finite sums and 
$C_{N}(K)$ is closed, we obtain

$$
\begin{aligned}
& \left(\int_{\mathbb{R}^{n}} d E, \int_{\mathbb{R}^{n}} x_{1} d E, \int_{\mathbb{R}^{n}} x_{2} d E, \ldots, \int_{\mathbb{R}^{n}} x_{n}^{d} d E\right) \\
= & \int_{\mathbb{R}^{n}} v(x) d E(x) \\
= & \sum_{i} A_{i} v_{i} \\
= & \sum_{i} A_{i} v\left(x_{i}\right) \\
= & \left(\sum_{i} A_{i}, \sum_{i} A_{i} x_{i 1}, \sum_{i} A_{i} x_{i 2}, \ldots, \sum_{i} A_{i} x_{i n}^{d}\right),
\end{aligned}
$$

which verifies a quadrature formula for all monomials in $P_{d}\left(\mathbb{R}^{n}\right)$ simultaneously. Hence, we complete the proof.

Notice that, by the proof of Theorem 1, we have

$$
\begin{aligned}
& C_{N}(K) \\
= & \left\{\int_{\mathbb{R}^{n}} v(x) d E(x) \mid E \text { is a semi-spectral measure in } \mathbb{R}^{n}\right. \text { with moments up to } \\
& \text { degree } d\} .
\end{aligned}
$$

Proof of Theorem 2. Let us recall a standard Bolzano-Weierstrass trick. Given a real sequence, there is a subsequence that either converges or diverges to $-\infty$ or $\infty$. In the proof, we are going to keep using this trick.

Let $E$ be a semi-spectral measure on $\mathbb{R}^{n}$, which admits all its moments of degree less than or equal to $2 d$. We denote by $E_{r}$ the semi-spectral measure $E$ restricted to the closed ball $B_{r}$ of radius $r$, centered at origin. In order to use the trick mentioned above, let us consider that $r$ is a positive integer only. By Theorem 1, there is a system of $N\left(=l^{2} N_{2 d}\left(\mathbb{R}^{n}\right)\right)$ points, $x_{j}(r) \in B_{r}$, and semi-positive definite matrices $A_{j}(r)$ such that

$$
\int_{B_{r}} p d E=\sum_{j=1}^{N} A_{j}(r) p\left(x_{j}(r)\right)
$$

for $p \in P_{2 d}\left(\mathbb{R}^{n}\right)$.

Let $C$ be a positive constant such that $\operatorname{Tr}\left(\int_{\mathbb{R}^{n}}\left|x^{\alpha}\right| d E\right) \leq C$ for all $|\alpha| \leq 2 d$. Hence,

$$
0 \leq \operatorname{Tr}\left(A_{j}(r)\right) \leq \operatorname{Tr}\left(\sum_{j=1}^{N} A_{j}(r)\right)=\operatorname{Tr}\left(\int_{B_{r}} d E\right) \leq \operatorname{Tr}\left(\int_{\mathbb{R}^{n}} d E\right) \leq C .
$$

Therefore, by passing to a subsequence in $r$, we can assume that the limits

$$
\lim _{r \rightarrow \infty} A_{j}(r)=A_{j}
$$

exist for all $1 \leq j \leq N$.

Let $x_{j i}(r)$ be the $i$-th coordinate of $x_{j}(r)$ in $\mathbb{R}^{n}$. By passing to a subsequence

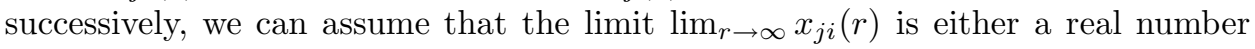
$x_{j i},-\infty$ or $\infty$ for all index pairs $(j, i), 1 \leq j \leq N$ and $1 \leq i \leq n$. 
Fix an index $j$. If $\lim _{r \rightarrow \infty} x_{j i}(r)=-\infty$ or $\infty$ for some index $i$, then

$$
0 \leq \operatorname{Tr}\left(A_{j}(r)\right) x_{j i}(r)^{2 d} \leq \operatorname{Tr}\left(\sum_{j=1}^{N} A_{j}(r) x_{j i}(r)^{2 d}\right)=\operatorname{Tr}\left(\int_{B_{r}} x_{j i}^{2 d} d E\right) \leq C,
$$

which implies that

$$
\lim _{r \rightarrow \infty} A_{j}(r)=0
$$

and

$$
\lim _{r \rightarrow \infty} \operatorname{Tr}\left(A_{j}(r)\right) x_{j i}(r)^{k}=0
$$

for $0 \leq k \leq 2 d-1$. Therefore, we obtain

$$
\lim _{r \rightarrow \infty}\left(\operatorname{Tr}\left(A_{j}(r)\right) \max _{1 \leq i \leq n}\left|x_{j i}(r)^{2 d-1}\right|\right)=0
$$

and

$$
0 \leq \lim _{r \rightarrow \infty} \operatorname{Tr}\left(A_{j}(r)\right)\left|x(r)^{\alpha}\right| \leq \lim _{r \rightarrow \infty}\left[\operatorname{Tr}\left(A_{j}(r)\right) \max _{1 \leq i \leq n}\left|x_{j i}(r)^{2 d-1}\right|\right]=0
$$

for $|\alpha| \leq 2 d-1$. Hence, we have

$$
\lim _{r \rightarrow \infty} A_{j}(r) x_{j}(r)^{\alpha}=0
$$

for $|\alpha| \leq 2 d-1$, i.e., we have proved

$$
\lim _{r \rightarrow \infty} A_{j}(r) p\left(x_{j}(r)\right)=0
$$

for $p \in P_{2 d-1}\left(\mathbb{R}^{n}\right)$.

Now, consider an index $j$ such that $\lim _{r \rightarrow \infty} x_{j i}(r)$ exists for every $i$. That is, $\lim _{r \rightarrow \infty} x_{j}(r)=x_{j}$ for some point $x_{j}$ in $\mathbb{R}^{n}$ (in the closed support of the measure). Hence we have

$$
\lim _{r \rightarrow \infty} A_{j}(r) p\left(x_{j}(r)\right)=A_{j} p\left(x_{j}\right) .
$$

Denote by $J$ the set of indices $j$ such that $\lim _{r \rightarrow \infty} x_{j}(r)$ exists. Therefore, we obtain

$$
\begin{aligned}
\int_{\mathbb{R}^{n}} p d E & =\lim _{r \rightarrow \infty} \int_{B_{r}} p d E \\
& =\lim _{r \rightarrow \infty} \sum_{j=1}^{N} A_{j}(r) p\left(x_{j}(r)\right) \\
& =\sum_{j \in J} A_{j} p\left(x_{j}\right)
\end{aligned}
$$

for $p \in P_{2 d-1}\left(\mathbb{R}^{n}\right)$ and complete the proof.

\section{Appendix}

For the convenience of readers who are not familiar with the trace norm, we give a brief introduction here. Let $V$ be the vector space of real symmetric $l \times l$ matrices. Let $\lambda_{1}, \lambda_{2}, \ldots, \lambda_{l}$ be the eigenvalues of a matrix $A \in V$. Define

$$
\tau(A) \equiv \sum_{i=1}^{l}\left|\lambda_{i}\right|
$$

Let $A$ represent a symmetric linear operator on $\mathbb{R}^{l}$, i.e., $\left\langle A y_{1}, y_{2}\right\rangle=\left\langle y_{1}, A y_{2}\right\rangle$ for all $y_{1}, y_{2} \in \mathbb{R}^{l}$. $\mathbb{R}^{l}$ has an orthonormal basis $\left\{\varphi_{i}\right\}$ consisting of eigenvectors of $A$ such that $A \varphi_{i}=\lambda_{i} \varphi_{i}$. 
Lemma 5. Let $A$ be a symmetric operator on $\mathbb{R}^{l}$. Then

$$
\sum_{i=1}^{l}\left|\lambda_{i}\right|=\sup _{\left\{\psi_{i}\right\}} \sum_{i=1}^{l}\left|\left\langle A \psi_{i}, \psi_{i}\right\rangle\right|
$$

where $\left\{\psi_{i}\right\}$ is an arbitrary orthonormal basis of $\mathbb{R}^{l}$.

Proof. $\left\{\varphi_{i}\right\}$ is an orthnormal basis of $\mathbb{R}^{l}$ and

$$
\sum_{i=1}^{l}\left|\left\langle A \varphi_{i}, \varphi_{i}\right\rangle\right|=\sum_{i=1}^{l}\left|\lambda_{i}\right|
$$

Hence

On the other hand, since

$$
\sum_{i=1}^{l}\left|\lambda_{i}\right| \leq \sup _{\left\{\psi_{i}\right\}} \sum_{i=1}^{l}\left|\left\langle A \psi_{i}, \psi_{i}\right\rangle\right|
$$

$$
\left\langle A \psi_{i}, \psi_{i}\right\rangle=\left\langle A \sum_{j}\left\langle\varphi_{j}, \psi_{i}\right\rangle \varphi_{j}, \psi_{i}\right\rangle=\sum_{j} \lambda_{j}\left\langle\varphi_{j}, \psi_{i}\right\rangle^{2}
$$

we have

$$
\sum_{i=1}^{l}\left|\left\langle A \psi_{i}, \psi_{i}\right\rangle\right| \leq \sum_{i=1}^{l} \sum_{j=1}^{l}\left|\lambda_{j}\right|\left\langle\varphi_{j}, \psi_{i}\right\rangle^{2}=\sum_{j=1}^{l}\left|\lambda_{j}\right|\left\|\varphi_{j}\right\|^{2}=\sum_{j=1}^{l}\left|\lambda_{j}\right| .
$$

Hence

$$
\sup _{\left\{\psi_{i}\right\}} \sum_{i=1}^{l}\left|\left\langle A \psi_{i}, \psi_{i}\right\rangle\right| \leq \sum_{j=1}^{l}\left|\lambda_{j}\right|
$$

Therefore,

$$
\sum_{i=1}^{l}\left|\lambda_{i}\right|=\sup _{\left\{\psi_{i}\right\}} \sum_{i=1}^{l}\left|\left\langle A \psi_{i}, \psi_{i}\right\rangle\right|
$$

In the following proposition, we prove that $\tau(A)$ is a norm on $V . \tau(A)$ is the so-called 'trace norm'.

Proposition 6. $\tau(A)$ is a norm on $V$.

Proof. It is obvious that $\tau(A) \geq 0$ and $\tau(a A)=|a| \tau(A)$ for $a \in \mathbb{R}$. If $\tau(A)=0, \lambda_{i}$ 's are zero. Hence, the characteristic polynomial of $A$ is $t^{l}$. Since $A$ is diagonalizable, the minimal polynomial $m(t)$ of $A$ has only simple roots. Hence $m(t)=t$ and $A=0$. Therefore, $\tau(A)=0$ if and only if $A=0$.

To complete the proof, we need to show that $\tau(A+B) \leq \tau(A)+\tau(B)$ for $A, B \in V$. Consider

$$
\sup _{\left\{\psi_{i}\right\}} \sum_{i=1}^{l}\left|\left\langle(A+B) \psi_{i}, \psi_{i}\right\rangle\right| \leq \sup _{\left\{\psi_{i}\right\}} \sum_{i=1}^{l}\left|\left\langle A \psi_{i}, \psi_{i}\right\rangle\right|+\sup _{\left\{\psi_{i}\right\}} \sum_{i=1}^{l}\left|\left\langle B \psi_{i}, \psi_{i}\right\rangle\right| .
$$

By the previous lemma, we obtain

$$
\tau(A+B) \leq \tau(A)+\tau(B) .
$$

Note that $\|A\|=\sup _{i}\left|\lambda_{i}\right| \leq \tau(A)$ and these two norms are equivalent. 


\section{ACKNOWLEDGEMENT}

The first author is grateful to M. Putinar for his guidance on this subject. We also want to thank the referee, whose suggestions improved our paper.

\section{REFERENCES}

[1] R. E. Curto and L. Fialkow, Flat extensions of positive moment matrices: relations in analytic or conjugate terms, Oper. Theory Adv. Appl., 104, 1998. MR 99i:47026

[2] S. Karlin and W. J. Studden, Tchebycheff Systems: With Applications in Analysis and Statistics, Pure and Applied Mathematics, Vol. XV, Interscience, 1966. MR 34:4757

[3] M. Putinar, A note on Tchakaloff's theorem, Proc. Amer. Math. Soc. 125 (1997), no. 8, 2409-2414. MR 97j:65045

[4] A. H. Stroud, Approximate Calculation of Multiple Integrals, Prentice-Hall, Englewood Cliffs, New Jersey, 1971. MR 48:5348

[5] V. Tchakaloff, Formules de cubature mécanique à coefficients non négatifs, Bull. Sci. Math. 81 (1957), 123-134. MR 20:1145

Department of Applied Mathematics, National Donghwa University, Shoufeng, HUALIEN 974, TAIWAN

E-mail address: lcwang@mail.ndhu.edu.tw

Institute of Statistics, National Chiao Tung University, Hsinchu 300, Taiwan

E-mail address: cchen@stat.nctu.edu.tw 